\title{
A COMMENT ON ENTROPY AND AREA*
}

\author{
D. Kabat and M. J. Strassler \\ Dept. of Physics and Astronomy \\ Rutgers University \\ Piscataway, NJ 08855-0849 \\ kabat,strasslr@physics.rutgers.edu
}

\begin{abstract}
For an arbitrary quantum field in flat space with a planar boundary, an entropy of entanglement, associated with correlations across the boundary, is present when the field is in its vacuum state. The vacuum state of the same quantum field appears thermal in Rindler space, with an associated thermal entropy. We show that the density matrices describing the two situations are identical, and therefore that the two entropies are equal. We comment on the generality and significance of this result, and make use of it in analyzing the area and cutoff dependence of the entropy. The equivalence of the density matrices leads us to speculate that a planar boundary in Minkowski space has a classical entropy given by the Bekenstein-Hawking formula.
\end{abstract}

hep-th/9401125

RU-94-10

January 1994

* This work is supported in part by funds provided by the U. S. Department of Energy (D.O.E.) under contract \#DE-FG05-90ER40559. 


\section{INTRODUCTION}

In a recent paper Srednicki ${ }^{1}$ considered the entropy of entanglement of a quantum field. Taking a free scalar field in its ground state, he calculated the density matrix which describes the state of the field outside an imaginary sphere when one chooses not to make measurements in the sphere's interior. He found numerically that the corresponding entropy scales as the surface area of the sphere. This entropy was originally studied by Bombelli et. al. ${ }^{2}$ as a quantum correction to the entropy of a black hole.

't Hooft has studied a seemingly different source of quantum corrections to the entropy of a black hole arising from scalar fields propagating in the region just outside the horizon ${ }^{3}$. For very massive black holes this region may be approximated as flat Rindler space, in which quantum fields are thermally excited at the Hawking-Unruh temperature and carry a corresponding thermal entropy. This entropy was likewise found to scale as the area of the horizon.

The purpose of this comment is to point out that, for a sphere of infinite radius, the density matrix studied by Srednicki is equal to the thermal density matrix describing the fields studied by 't Hooft. (This is a special case of a more general result proven in the next section.) It follows that the two entropies are identical in this limit and should be thought of as the same quantity measured by different classes of observers. The possibility of this equivalence was suggested by Bombelli et. al. ${ }^{2}$. Unfortunately the entropy is divergent; our discussion is therefore limited to its dependence on the area of the boundary and the degree of its divergence. Except in 1+1 dimensions, the coefficient of the divergence is non-universal. We will explain below that, as a result of subtleties involving regulators, Srednicki's actual computation $^{1}$ cannot be quantitatively compared with any of 't Hooft's results. ${ }^{3}$

Other groups are investigating these issues and have reached similar conclusions ${ }^{4,5,6}$. 


\section{PROOF OF EQUIVALENCE}

In this section we show that for the vacuum state of a field theory, the density matrix found by tracing over the fields in half of space is equal to the thermal density matrix describing the fields in Rindler space. This result holds for a wide class of field theories; we will discuss its generality in the next section.

Consider a field $\phi\left(t, x, y_{1}, \ldots, y_{d_{\perp}}\right)$ in a $\left(d_{\perp}+1\right)+1$ dimensional Minkowski space. We will describe the field configuration $\phi\left(t, x, y_{i}\right)$ as $\phi_{R}\left(t, x, y_{i}\right)$ for $x>0$ and as $\phi_{L}\left(t, x, y_{i}\right)$ for $x<0$; in subsequent expressions we suppress the dependence on the variables $y_{i}$. At a fixed time $t_{0}$ take the field to be in its ground state, with density matrix $|0><0|$, and form the density matrix $\rho_{R}$ describing the state of the field $\phi_{R}\left(t_{0}, x\right)$ for $x>0$ by tracing over all degrees of freedom $\phi_{L}\left(t_{0}, x\right)$ located at $x<0$.

$$
<\phi_{R}^{\prime}\left|\rho_{R}\right| \phi_{R}^{\prime \prime}>=\int \mathcal{D} \phi_{L}<\phi_{L} \phi_{R}^{\prime}|0><0| \phi_{L} \phi_{R}^{\prime \prime}>
$$

The entropy of entanglement is defined to be

$$
S=-\int \mathcal{D} \phi_{R}<\phi_{R}\left|\rho_{R} \log \rho_{R}\right| \phi_{R}>.
$$

To show that this entropy is thermal, we introduce a Euclidean path integral representation for the density matrix (1). First, we use a Euclidean functional integral to generate projections onto the vacuum.

$$
<0 \mid \phi_{L}^{\prime} \phi_{R}^{\prime}>=\int \mathcal{D} \phi \exp \left[-\int_{0}^{\infty} d \tau \int d x d^{d_{\perp}} y \mathcal{L}\right]
$$

The integral is over all fields $\phi(\tau>0, x)$ that at $\tau=0$ take on the value $\phi_{L}^{\prime}(x)$ for $x<0$ and $\phi_{R}^{\prime}(x)$ for $x>0$. Putting two of these functional integrals together and integrating over $\phi_{L}^{\prime}(x)$ 
leads to a representation of the density matrix element (1) as a single Euclidean functional integral on a space with a cut at the set of points $\{\tau=0, x>0\}$, subject to the boundary condition that $\phi(\tau=+\epsilon, x>0)=\phi_{R}^{\prime \prime}(x)$ just above the cut and $\phi(\tau=-\epsilon, x>0)=\phi_{R}^{\prime}(x)$ just below it,

$$
\begin{gathered}
<\phi_{R}^{\prime}\left|\rho_{R}\right| \phi_{R}^{\prime \prime}>=C \int \mathcal{D} \phi e^{-S} \delta\left[\phi_{R}(\tau=-\epsilon)-\phi_{R}^{\prime}\right] \delta\left[\phi_{R}(\tau=+\epsilon)-\phi_{R}^{\prime \prime}\right] \\
\exp \left\{+\int_{-\epsilon}^{+\epsilon} d \tau \int_{0}^{\infty} d x \int d^{d_{\perp}} y \mathcal{L}\right\} .
\end{gathered}
$$

Here $\epsilon$ is infinitesimal and $C$ is chosen to normalize $\operatorname{Tr} \rho_{R}=1$.

Now think of this path integral as generating time evolution under some Hamiltonian. In this geometry, instead of taking the usual Hamiltonian and time slices at fixed $\tau$, it is natural to use angular quantization ${ }^{7}$. Introduce the Euclidean Rindler Hamiltonian $H_{R}$ which is the generator of rotations in the $\tau-x$ plane (in Minkowski space it is the Lorentz boost generator). The above path integral is then seen to be $<\phi_{R}^{\prime}\left|\exp \left(-2 \pi H_{R}\right)\right| \phi_{R}^{\prime \prime}>$, so $\rho_{R}=e^{-2 \pi H_{R}}$. $^{*}$ This completes the formal proof that the density matrix in half of Minkowski space is a thermal ensemble with respect to the Rindler Hamiltonian, with an inverse temperature $\beta=2 \pi$. **

\section{DISCUSSION}

This equivalence is very satisfying: the two seemingly different density matrices are actually the same density matrix interpreted by different observers. An inertial observer

* This is nothing more than the standard Euclidean demonstration that the Minkowski vacuum looks thermal to a Rindler observer.

** The statement that the density matrix is thermal requires clarification. Any density matrix has a well defined logarithm, so $\rho=e^{-H}$ for some $H$. This case is special in that $H$ is the simple local operator $H_{R}$. 
who chooses not to make measurements on the field at $x<0$ obtains exactly the same results for any experiment as a Rindler observer who is prevented by a horizon from making measurements at $x<0$. In particular the two entropies are formally identical, which is a great technical simplification, since the entropy of entanglement defined in (2) is a difficult object to compute, while thermodynamics in Rindler space is relatively straightforward.

A few comments are in order about the generality of our proof. No assumptions are made about the field theory, except that it must be local and must possess Poincaré invariance in the $x-t$ plane; the theory need not be free. The proof requires that the field be in its vacuum state, and does not apply if some other state is chosen. It is also specific to the geometry which we use; the equivalence is exact only for spaces which are divided in half by a planar boundary. It gives a good approximation to the density matrix which Srednicki considered ${ }^{1}$, for which the excluded region is a finite sphere, as long as the radius of the sphere is large. Finite radius corrections are especially small for the entropy of entanglement, which, as we will show, comes predominantly from short-wavelength modes. For example, if the theory of a scalar field were modified with a physical cutoff at the Planck scale, making the entropy finite, then, for any sphere of radius much larger than the Planck length, the entropy of entanglement outside the sphere would equal the thermal entropy outside a black hole of the same radius. Directly analyzing the entropy of entanglement in more general geometries requires working with the definition (2); for developments in this direction see the work of Callan and Wilczek ${ }^{4}$.

To understand the physical basis for the equivalence of the two density matrices, we focus attention on the quadrant $Q$ of Minkowski space with $x>|t|$, since $Q$ is the part of spacetime causally disconnected from the $t=0, x<0$ half-space. Rindler and inertial observers have 
different ways of restricting their measurements to $Q$. A Rindler observer at a proper distance $s$ from the horizon $x=|t|$ remains within $Q$ by accelerating with a proper acceleration $1 / s ;$ consequently the observer experiences a local temperature $1 / 2 \pi s$. An inertial observer, whose world line does not remain within $Q$, must take a different approach. Consider an experiment performed by an inertial observer at a distance $x_{0}$ from the imaginary boundary at $x=0$. From the moment the experiment begins, its effects begin to propagate from $x=x_{0}$ to $x=0$; given sufficient time they will interact with the $x<0$ half-space and return to $x=x_{0}$, allowing the $x<0$ region to affect the results of the experiment. The experiment must therefore be completed in a time $\Delta t<2 x_{0}$, which keeps it strictly within $Q$; this leads to energy fluctuations of order $1 / 2 x_{0}$, comparable to the temperature experienced by Rindler observers at the proper distance $s=x_{0}$. This highlights the connection between ordinary quantum fluctuations and the local temperature of Rindler space, and clarifies how the same density matrix can describe both situations.

Since the density matrices are equivalent, one may compute any observable at $t=0$ either with the reduced density matrix (1) or with thermodynamics in Rindler space; the results will be identical. It follows that the half-space entropy of entanglement is formally equal to the Rindler space entropy. However, both entropies are divergent in most quantum field theories, and it seems unlikely that any observable exists which would allow them to be directly compared. Despite this, we will consider, for a free scalar field, the finite thermal entropy contained in a box in Rindler space. This quantity is adequate to study the universal divergence and scaling properties of the entropy of entanglement. The coefficient of the divergence is not universal, except in $1+1$ dimensions, and cannot be directly compared with Srednicki's coefficient. 


\section{ENTROPY CALCULATIONS}

We now present a few calculations of the Rindler entropy in a box, following the original work of 't Hooft ${ }^{3}$. These results will allow us, using the equivalence proved above, to find the area dependence and degree of divergence of the entropy of entanglement. As we will see, naive scaling analysis would have given the correct answer ${ }^{1,2}$. In more than two dimensions the coefficient of the divergence is non-universal.

We begin in $1+1$ dimensions. Let us compute at a fixed time the entropy contained in a box with walls at $x=x_{0}$ and $x=x_{1}{ }^{*}$ the coordinate $x$ measures proper distance from the Rindler horizon. For a well-defined counting of the number of modes within the box boundary conditions must be chosen at $x_{0}$ and $x_{1}$; some of our results are sensitive to this choice. We work in the semiclassical (WKB) approximation; for a given (dimensionless) Rindler energy $E$ the accumulated phase of the wave function is given by

$$
(n(E)+\alpha) \pi=\int_{x_{0}}^{x_{\max }} \frac{d x}{x} \sqrt{E^{2}-m^{2} x^{2}} .
$$

This follows from adapting equation (3.7) of 't $\mathrm{Hooft}^{3}$ for the case of large black hole mass. The upper limit of integration $x_{\max }$ is taken to be the smaller of the large distance cutoff $x_{1}$ and the point $E / m$ at which the integrand vanishes. The WKB quantization condition states that for each $E$ such that $n(E)$ is an integer $j \geq 0$, there is a state with energy $E_{j}=E$. The constant $\alpha$ is to be chosen according to the relevant boundary conditions. If the upper limit of integration is set by $x_{1}$ and the boundary conditions are $\phi\left(x_{0}\right)=\phi\left(x_{1}\right)=0$, then $\alpha=1$;

$* x_{0}$ and $x_{1}$ bound the region over which a given experiment to measure the entropy is sensitive. They are not regulators for the field theory; in fact we perform the whole calculation in the continuum. 
if the upper limit of integration is set by $E / m$ then $\alpha=3 / 4$ for $\phi\left(x_{0}\right)=0$ and $\alpha=1 / 4$ for $\phi^{\prime}\left(x_{0}\right)=0$. The WKB approximation should be quite accurate in this potential, even for low lying energy levels ${ }^{8}$.

The free energy is given by a sum over energy eigenstates

$$
\beta F=\sum_{j} \log \left(1-e^{-\beta E_{j}}\right)
$$

the entropy in Rindler space is $S=\left(\beta \frac{\partial}{\partial \beta}-1\right)(\beta F)$ with $\beta$ set equal to $2 \pi$. For small $\beta m x_{0}$ the sum over states in the partition function is dominated by highly excited states, so we may introduce a smoothed density of states $g(E) \equiv \frac{d n(E)}{d E}$ and replace $\sum_{j} \rightarrow \int d E g(E)$. Note that we shall not see any dependence on the choice of boundary conditions, as the density of states is insensitive to the value of $\alpha$ in (3). Integrating by parts gives

$$
\beta F=-\beta \int d E \frac{n(E)}{e^{\beta E}-1}
$$

with the convention $n(E)=0$ for $E<m x_{0}$. Expanding the Bose-Einstein distribution in powers of $e^{-\beta E}$ and performing first the $E$ integration, then the $x$ integration, gives

$$
\beta F=\frac{1}{\pi \beta} \sum_{n=1}^{\infty} \frac{1}{n^{2}}\left[K_{0}\left(n \beta m x_{1}\right)-K_{0}\left(n \beta m x_{0}\right)\right]
$$

In appendix A we show that these sums behave as

$$
\sum_{n=1}^{\infty} \frac{1}{n^{2}} K_{0}(n t) \sim-\frac{\pi^{2}}{6} \log t+\text { const. } \quad t \ll 1 .
$$

This gives the leading behavior of the free energy $\beta F$ in the regimes

$$
\begin{array}{ll}
x_{0} \ll x_{1} \ll \frac{1}{m}: & -\frac{1}{\pi \beta} \frac{\pi^{2}}{6} \log \frac{x_{1}}{x_{0}}+\mathcal{O}\left(m x_{1}\right) \\
x_{0} \ll \frac{1}{m} \ll x_{1}: & -\frac{1}{\pi \beta}\left[\frac{\pi^{2}}{6} \log \frac{1}{\beta m x_{0}}+\mathcal{O}(1)\right]
\end{array}
$$


with corresponding entropies

$$
\begin{array}{ll}
x_{0} \ll x_{1} \ll \frac{1}{m}: & \frac{1}{6} \log \frac{x_{1}}{x_{0}}+\mathcal{O}\left(m x_{1}\right) ; \\
x_{0} \ll \frac{1}{m} \ll x_{1}: & \frac{1}{6} \log \frac{1}{m x_{0}}+\mathcal{O}(1) .
\end{array}
$$

The first expression matches the exact $m=0$ result obtained in conformal field theory ${ }^{4,5}$. In the second, intermediate mass case, we see that the mass takes over from $x_{1}$ in setting a large distance cutoff.

We now consider the behavior in $1+1$ dimensions for $\beta m x_{0}>1$. Since $\beta E_{j} \geq \beta m x_{0}$ we may set

$$
\beta F \approx-\sum_{j} e^{-\beta E_{j}} .
$$

In this case only the lowest lying modes in the box are occupied, and it is inappropriate to replace the sum over energy levels with an integral. The choice of boundary conditions is quite important now; for $\phi\left(x_{0}\right)=0$

$$
\beta F=-e^{-\beta m x_{0}}\left(e^{-1.84 \beta\left(m x_{0}\right)^{1 / 3}}+e^{-3.24 \beta\left(m x_{0}\right)^{1 / 3}}+\cdots\right)
$$

while for $\phi^{\prime}\left(x_{0}\right)=0$

$$
\beta F=-e^{-\beta m x_{0}}\left(e^{-0.89 \beta\left(m x_{0}\right)^{1 / 3}}+e^{-2.59 \beta\left(m x_{0}\right)^{1 / 3}}+\cdots\right)
$$

These energy levels are found by solving the WKB quantization condition (3) in the limit of large $m x_{0}$. As expected, the free energy is exponentially suppressed. If the sum over states were incorrectly approximated with an integral over $E$, the calculation would again lead to (4), which in the limit of large mass shows the correct $e^{-\beta m x_{0}}$ suppression but misses entirely the subleading $e^{-\beta\left(m x_{0}\right)^{1 / 3}}$ factors. We will see this issue is relevant for the higher dimensional case. 
To discuss the entropy in higher dimensions, we use a box extending from $x=x_{0}$ to $x=x_{1}$, with sides of length $L$ in each of the $d_{\perp}$ transverse dimensions. We consider massless fields; finite mass effects are presented in appendix B. The free energy $\beta F$ can be expressed as a sum over transverse modes labeled by their transverse momentum $k_{\perp}$. Each mode is equivalent to a $1+1$ dimensional scalar field with an effective mass $m_{\mathrm{eff}}=\left|k_{\perp}\right|$, whose free energy $\beta F_{1+1}\left(m_{\mathrm{eff}}, x_{0}, x_{1}, \beta\right)$ we have already computed.

$$
\beta F=\sum_{k_{\perp}} \beta F_{1+1}\left(\left|k_{\perp}\right|, x_{0}, x_{1}, \beta\right)
$$

Because a long distance cutoff in the longitudinal direction is no longer needed for a finite result, we can set $x_{1}=\infty$; in this limit, by dimensional analysis, $F_{1+1}$ is a function only of $\left|k_{\perp}\right| x_{0}$ and $\beta$. Approximating the sum as an integral $(L / 2 \pi)^{d_{\perp}} \int d^{d_{\perp}} k_{\perp}$ and changing variables to $\kappa=k_{\perp} x_{0}$, we have

$$
\beta F=\left(\frac{L}{2 \pi x_{0}}\right)^{d_{\perp}} \int d^{d_{\perp}} \kappa \beta F_{1+1}(|\kappa|, \beta)
$$

This is of the form $\beta F=C_{d_{\perp}}(\beta)\left(\frac{L}{x_{0}}\right)^{d_{\perp}}$, where $C_{d_{\perp}}(\beta)$ is a dimension dependent function of $\beta$.

We now check that no important $L$ dependence was hidden when the sum over $k_{\perp}$ was replaced by an integral. (By dimensional analysis this will also ensure there is no hidden $x_{0}$ dependence.) When $|\kappa| \equiv\left|k_{\perp}\right| x_{0} \sim x_{0} / L$, the integral should really be treated as a sum, whose details will depend on the boundary conditions on the walls of the transverse box. We can estimate the importance of this effect by computing the contribution to the free energy from the part of the integration region up to $|\kappa|=c x_{0} / L$, for $c$ of order one.

$$
\left(\frac{L}{2 \pi x_{0}}\right)^{d_{\perp}} \int_{|\kappa| \leq \frac{c x_{0}}{L}} d^{d_{\perp}} \kappa \frac{\pi}{6 \beta} \log \beta|\kappa| \sim \log \left(\beta c x_{0} / L\right) .
$$


Relative to the $\left(L / x_{0}\right)^{d_{\perp}}$ dependence of the leading term in (6), this is negligible. We therefore conclude that the straightforward scaling analysis is correct ${ }^{1,2}$ : in any dimension $d_{\perp}>0$ both the free energy and the entropy are proportional to the area $L^{d_{\perp}}$ of the transverse box, and have a $d_{\perp}$-th order power-law divergence $1 / x_{0}^{d_{\perp}}$ as the near edge of the box approaches the boundary $\left(x_{0} \rightarrow 0\right)$.

The coefficient

$$
C_{d_{\perp}}(\beta)=\left(\frac{1}{2 \pi}\right)^{d_{\perp}} \int d^{d_{\perp}} \kappa \beta F_{1+1}(|\kappa|, \beta)
$$

gets its largest contribution from modes with $|\kappa|$ of order $1 / \beta$. The function $\beta F_{1+1}(|\kappa| \equiv$ $\left.m_{\mathrm{eff}} x_{0}, \beta\right)$ increases logarithmically until $|\kappa| \sim 1 / \beta$, then decreases exponentially; additional powers of $|\kappa|$ in the integration measure push the main contribution to $C_{d_{\perp}}(\beta)$ out somewhat further. As discussed above, for $\beta m_{\mathrm{eff}} x_{0} \geq 1$ it is a poor approximation to replace the sum over states with an integral over a smoothed density of states. The result ' $t$ Hooft obtained ${ }^{3}$ for $C_{2}(\beta)$ may not be reliable for this reason. One can in principle determine $C_{d_{\perp}}(\beta)$ exactly, for some choice of boundary conditions, by solving the Schrödinger equation to obtain the spectrum of states; this probably cannot be done analytically. However, since a mode with large effective mass has a free energy which is quite sensitive to the boundary conditions at $x=x_{0}$, different choices for the boundary conditions will lead to different values of $C_{d_{\perp}}(\beta)$. This implies that $C_{d_{\perp}}(\beta)$ is not a universal quantity.

Even if the coefficient $C_{d_{\perp}}(\beta)$ were exactly known for a particular set of boundary conditions, it would be impossible to compare it quantitatively with Srednicki's results. Srednicki computes the entropy in a region $x_{0}<x<x_{1}$ with $x_{0}=a / 2$, where $a$ is the spacing of the longitudinal lattice which he introduces as a regulator. (By "longitudinal" we refer to the direction perpendicular to the surface of his sphere.) In the continuum limit $a \rightarrow 0, x_{0} \rightarrow 0$ 
as well, and the entropy in his region becomes infinite. This quantity allows him to correctly study the scaling and the divergence structure of the entropy, but since his observable is not defined in the continuum, our proof cannot be used to relate his result directly to any finite computation in Rindler space. To see the connection with Rindler space one must define a measurable quantity which is finite in the continuum limit; the physical observable must be separated from the regulator of the field theory.

\section{CONCLUSIONS}

We briefly review our main points.

A simple proof demonstrates that the density matrix of a quantum field theory obtained in the vacuum state by tracing over half of space is identical to the thermal density matrix of the field in Rindler space. This holds for any local, renormalizable theory which is Poincaré invariant in the $x-t$ plane. We discuss the physical basis for this equivalence. It follows that the entropies studied by Srednicki ${ }^{1}$ and by 't Hooft ${ }^{3}$ are formally equal. Unfortunately, with few known exceptions ${ }^{6}$, these entropies are infinite, in which case it is probably impossible to define a physically sensible and finite observable which can be used to directly compare them.

Using the formal equivalence of the entropies, we argue that the entropy of entanglement for a free scalar field is logarithmically divergent in $1+1$ dimensions and $d_{\perp}$-order divergent in $\left(d_{\perp}+1\right)+1$ dimensions. The normalization of the entropy is universal in $1+1$ dimensions and non-universal otherwise. We also show that for $d_{\perp}>0$ the entropy of entanglement is proportional to the area of the boundary.

We conclude by noting an important possible implication of the equivalence of the two density matrices. Both Srednicki and 't Hooft compute entropies associated with one-loop 
effects of scalar fields in a background metric. In the presence of general relativity, there should be a tree-level contribution to both entropies. In other words, corresponding to the Bekenstein-Hawking entropy of the horizon of an infinitely massive black hole, there should be an entropy associated with an imaginary planar boundary in perfectly flat space, with $S=($ Area $) / 4$. If this boundary entropy ought to be interpreted as a classical entropy of entanglement, then it suggests that whatever the fundamental generalization of Einstein's theory of gravity, it should be non-local at short distances, even at the classical level. In particular, Susskind and collaborators ${ }^{6}$ have suggested that in string theory this entropy is associated with strings which classically straddle the boundary.

While this manuscript was in preparation, results related to those presented here appeared in refs. 4 and 6.

\section{ACKNOWLEDGEMENTS}

We thank Tom Banks and Stephen Shenker for their many valuable contributions to this paper, and Leonard Susskind and Frank Wilczek for interesting discussions.

\section{APPENDIX A: SUMS}

We consider sums of the form

$$
f(t)=\sum_{n=1}^{\infty} \frac{1}{n^{2}} K_{0}(n t)
$$

where $K_{0}$ is a modified Bessel function. To extract the behavior for small $t$ we calculate the Mellin transformation ${ }^{9}$

$$
\begin{aligned}
F(\xi) & \equiv \int_{0}^{\infty} d t t^{\xi-1} f(t) \\
& =2^{\xi-2} \zeta(2+\xi)\left[\Gamma\left(\frac{\xi}{2}\right)\right]^{2} .
\end{aligned}
$$


Suppose $F(\xi)$ has poles at $\xi_{1}, \xi_{2}, \ldots$, and write the principle part of the Laurent expansion of about the $i^{\text {th }}$ pole

$$
F(\xi)=\frac{b_{1 i}}{\xi-\xi_{i}}+\frac{b_{2 i}}{\left(\xi-\xi_{i}\right)^{2}}+\cdots
$$

Then asymptotically for small $t$

$$
f(t)=\sum_{i} t^{-\xi_{i}}\left(b_{1 i}+\frac{1}{1 !} b_{2 i}(-\log t)+\frac{1}{2 !} b_{3 i}(-\log t)^{2}+\cdots\right)
$$

The sum is dominated by the double pole from the gamma functions at $\xi=0$, with subleading behavior from the simple pole in the zeta function at $\xi=-1$. One finds

$$
f(t) \sim-\frac{\pi^{2}}{6} \log t+\frac{\pi^{2}}{6}(\log 2-\gamma)+\zeta^{\prime}(2)+\mathcal{O}(t)
$$

For large $t, f(t)$ is exponentially suppressed.

\section{APPENDIX B: FINITE MASS}

The free energy of a scalar field of mass $m, 1 / L \ll m \ll 1 / x_{0}$, is equal to the massless free energy (6) plus a correction

$$
\beta F_{m \neq 0}-\beta F_{m=0}=\left(\frac{L}{2 \pi x_{0}}\right)^{d_{\perp}} \int d^{d_{\perp}} \kappa\left[\beta F_{1+1}\left(\sqrt{\kappa^{2}+m^{2} x_{0}^{2}}, \beta\right)-\beta F_{1+1}(|\kappa|, \beta)\right] .
$$

For $d_{\perp} \leq 2$ the main contribution comes from the infrared, $|\kappa| \sim m x_{0}$, where the behavior of the integrand is known from our $1+1$ dimensional calculations.

$$
\beta F_{m \neq 0}-\beta F_{m=0} \approx\left(\frac{L}{2 \pi x_{0}}\right)^{d_{\perp}} \frac{\pi}{6 \beta} \int d^{d_{\perp}} \kappa \log \left[1+\left(m x_{0} / \kappa\right)^{2}\right]
$$

This gives the corrections

$$
\begin{aligned}
& \frac{\pi}{12} \frac{L m}{\beta} \quad\left(d_{\perp}=1\right) ; \\
& -\frac{1}{24} \frac{L^{2} m^{2}}{\beta} \log m x_{0} \quad\left(d_{\perp}=2\right) .
\end{aligned}
$$


Since these corrections are finite and logarithmically divergent, respectively, and come from a region of the integral where the integrand is insensitive to boundary conditions, we conjecture that their coefficients are universal. For $d_{\perp}>2$ the integral is dominated by $|\kappa| \gg m x_{0}$, where we may expand the integrand in powers of $m$.

$$
\begin{aligned}
\beta F_{m \neq 0}-\beta F_{m=0} & \approx\left(\frac{L}{2 \pi x_{0}}\right)^{d_{\perp}} \int d^{d_{\perp}} \kappa\left(m x_{0}\right)^{2} \frac{d}{d \kappa^{2}}\left[\beta F_{1+1}(|\kappa|, \beta)\right] \\
& =-\frac{\left(d_{\perp}-2\right) \pi^{d_{\perp} / 2}}{\Gamma\left(d_{\perp} / 2\right)}\left(m x_{0}\right)^{2}\left(\frac{L}{2 \pi x_{0}}\right)^{d_{\perp}} \int d^{d_{\perp}} \kappa \kappa^{d_{\perp}-3}\left[\beta F_{1+1}(|\kappa|, \beta)\right] \\
& =-\left.\frac{1}{4 \pi}(m L)^{2} \beta F\right|_{\left(d_{\perp}-2\right)}
\end{aligned}
$$

This is proportional to the free energy in $d_{\perp}-2$ dimensions, which is power-law divergent as $x_{0} \rightarrow 0$ and depends on boundary conditions, and so this correction is non-universal; still this result is rather interesting.

\section{REFERENCES}

1. M. Srednicki, Phys. Rev. Lett. 71, 666 (1993).

2. L. Bombelli, R. K. Koul, J. Lee, and R. D. Sorkin, Phys. Rev. D34, 373 (1986). See in particular footnote 17 .

3. G. 't Hooft, Nucl. Phys. B256, 727 (1985).

4. C. Callan and F. Wilczek, On Geometric Entropy, preprint IASSNS-HEP-93/87, hepth/9401072 (January 1994).

5. C. Holzhey, Princeton University thesis (unpublished, 1993); C. Holzhey, F. Larsen, and F. Wilczek, Geometric Entropy in Conformal Field Theory, preprint IASSNS-HEP $93 / 88$.

6. L. Susskind and J. Uglum, Black Hole Entropy in Canonical Quantum Gravity and Superstring Theory, Stanford preprint SU-TP-94-1, hep-th/9401070 (January 1994); 
L. Susskind, Some Speculations about Black Hole Entropy in String Theory, preprint RU-93-44, hep-th/9309145 (September 1993).

7. The quantum theories resulting from the different choices of time slicing will be identical. D. G. Boulware, Phys. Rev. D11, 1404 (1975).

8. J. J. Sakurai, Modern Quantum Mechanics (Addison-Wesley, 1985), p. 108.

9. F. Oberhettinger, Tables of Mellin Transforms (Springer-Verlag, 1974), p. 7. 